\title{
High-Throughput Screening of Molecular Catalysts Using Automated Liquid Handling, Injection, and Microdevices
}

\author{
Claude de Bellefon*, Radwan Abdallah, Thierry Lamouille, Nathalie Pestre, Sylvain Caravieilhes, \\ and Pierre Grenouillet
}

\begin{abstract}
A new concept for the high-throughput screening of multiphase reactions involving molecular catalysts is described. It combines pulse injections of catalysts and substrate and mixing in a microdevice. The novelty of the concept resides in dynamic sequential vs. batchwise operations. Some advantages over traditional batch parallel operations are lower inventory of sample (down to $\mu \mathrm{g}$ ) coupled with accurate control of reaction time, larger range of operating conditions (pressure, temperature), simpler and fewer electromechanical moving parts and easy automation. Results on liquid-liquid (biphasic allylic alcohol isomerization), and gas-liquid (asymmetric hydrogenation) reactions indicates that the number of tests per day can easily reach $50 \mathrm{~d}^{-1}$ with inventory of sample $(\mathrm{Rh})$ per test as low as $5 \mathrm{\mu g}$.
\end{abstract}

Keywords: Asymmetric hydrogenation · High-throughput screening · Homogeneous catalysis · Kinetics · Microreactor

\section{Introduction}

The interest in methods to speed-up discovery in many fields of chemistry has been steadily increasing since the early 1990s. Many efforts have been put into catalyst discovery, mostly in the field of heterogeneous catalysis [1], more precisely, for gas-solid processes. Catalysis by organometallic compounds is also important for the large-scale production of polymers and many $\mathrm{C}-\mathrm{C}$ bond forming reactions such as hydroformylation, hydrocyanation, carbonylation, to name a few [2]. Metallocene compounds used as new catalysts for olefin polymerization encompass an extraordinary range of structural diversity and are the target of fast investigation methods. Besides polymer chemistry, transition metal

Correspondence: Dr. C. de Bellefon Laboratoire Génie des Procédés Catalytiques CNRS/ESCPE Lyon

F-69616 Villeurbanne, France

Tel.: +33472 431754

Fax: +33472431673

E-Mail: cdb@lgpc.cpe.fr complexes are also used for the small-scale production of high-value-added (chiral) molecules, for the pharma, agro, and fragrance industries. Again, chemical diversity is a key word since it is generally admitted that in the view of the diversity of the substrates (intermediates), there is no generic catalyst that will fit the activity/selectivity(enantioselectivity) required. This may be called the substrate/catalyst specificity and is well demonstrated in the field of asymmetric hydrogenation [3]. Many reports, from academia and industry, describe the application of fast investigation methods, albeit generally limited to slow ( $>1 \mathrm{~h}$ ) and monophasic (liquid) reactions [4]. Leading or younger high-tech companies such as Symyx, Avantium and HTE have built their business on what is nowadays called high-throughput screening (HTS), experimentation (HTE) or investigation (HTI) for catalysis and most of the major players in the chemical industries have setup HTS teams for catalysts discovery and catalytic processes optimization.

Is there a life behind this picture? The following is an attempt to provide both an analysis of what is actually required for HTS reactors as well as to disclose some recent results from our research aimed at the use of microstructures for HTS.

\section{Requirements for HTS of Catalytic Reactions}

Traditional reactors for the investigation of multiphase reactions should fulfill at least five requirements: i) multiphase capabilities, ii) flexible residence time, iii) flexible range of operating conditions, iv) chemical compatibility, v) no mass and heat transfer limitations and vi) ideal hydrodynamics [5].

The three first requirements are easily understood. A reactor must be designed to allow the contact between the several phases, gas-solid, gas-liquid (G/L), gasliquid-solid and liquid-liquid (L/L). It is clear for example in the case of a solidliquid reaction that the solid must be ideally dispersed in the reaction volume. A reactor in which the solid decants would seldom be classified as a good reactor. A flexible residence (reaction) time, the range of operating conditions and the material used for the reactor are also generally taken care of when choosing a test reactor. 
However, considerations are rarely given to mass transfer problems and non-ideal hydrodynamics. For example, one may easily find the range of pressure and temperature as well as the building material for labtop pressure reactors but no data concerning, for example, gas to liquid mass transfer capabilities are provided (except for the agitation speed), the same being true for micromixing. Mass and heat transfer limitations have been evidenced for many gas-solid and gas-liquid-solid catalytic reactions and chemists in these fields are well aware of such problems. In the field of gas-liquid (G/L) catalysis, i.e. homogeneous catalysis, the background of researchers tends to be in synthetic chemistry. They feel themselves generally much less concerned with such a 'chemical engineering approach' and believe their chemistry to be free of such phenomena. Several pieces of evidence however demonstrate the importance of such phenomena, particularly in the field of asymmetric hydrogenation. While the mechanism by which $\mathrm{G} / \mathrm{L}$ mass transfer limitation can affect the enantioselectivity has been analyzed in detail [6], it is true that the generality of such a coupling remains to be demonstrated through proper experimentation.

Three more requirements must be considered for high-throughput experimentation for catalytic reactions: a) sample (catalyst) quantities used for one test must de- crease from milligrams down to micrograms, while providing the same quality of data, b) the number of tests per time unit must be significantly higher than conventional techniques, i.e. the throughput testing frequency (TTF) must ideally be more than $\left.100 \mathrm{~d}^{-1}, \mathrm{c}\right)$ the HTS apparatus must be able to operate unattended, i.e. automation (sampling etc.) should be easy, fast and not too expensive.

Micro devices seem to be the ideal tool to answer these requirements. However, while many applications of micro-structured reactors have been found for laboratory research and small-scale productions, very few concern high-throughput experimentation for catalysis and almost none for organometallic catalysis [7]. Commercial apparatus that may be used for highthroughput (fast) screening of multiphase catalytic reactions are in fact all based on a very simple concept (Fig. 1, left): several bench-top batch-type tank reactors are downscaled (step i) and placed in a rack (step ii). Ideally, one reaction/catalyst or substrate can thus be screened in each of these minireactors (step iii) [8].

A new concept for high-throughput experiments that is based on dynamic sequential operations with a combination of pulse injection and micromachined elements has been proposed (Fig. 1, right) [9]. Two carrier fluids $(\mathrm{L} / \mathrm{L}$ or $\mathrm{G} / \mathrm{L})$ are circulated in a tube that ensures contact. A mixing unit $(\mu)$ is then placed at the entrance of the tube in order to increase the contact area thus preventing mass transfer limitations. In a further step, pulses of reagents and catalysts are introduced. The mixer will generate a well-mixed segment which will travel through the tube whose length determines the residence time. At the outlet of the system, the pulse is collected and analyzed for activity and selectivity. For HTS, sequential injections of other catalysts/substrates may be performed in a short time. The limiting time will be that required to avoid overlap of the reacting segments all along the tube reactor, as demonstrated by the residence time distribution [10]. When compared, the advantages of this new concept are easily understood. While in Fig. 1, left, each test requires one physical (stainless steel) reactor, it is only a pulse in Fig. 1, right, the same physical reactor being used sequentially for many diversities. Thus, some expected advantages over traditional batch parallel operations are a lower inventory of sample (down to $\mu \mathrm{g}$ ), a larger range of operating conditions (pressure, temperature), simpler and fewer electro-mechanical moving parts.

This concept covers a broad range of reaction types: monophasic fast reactions where mixing is important and which cannot be run in microtitration plates (stop-flow investigations); $\mathrm{L} / \mathrm{L}$ and $\mathrm{G} / \mathrm{L}$ reactions where mass transfer might hide the chemical features.

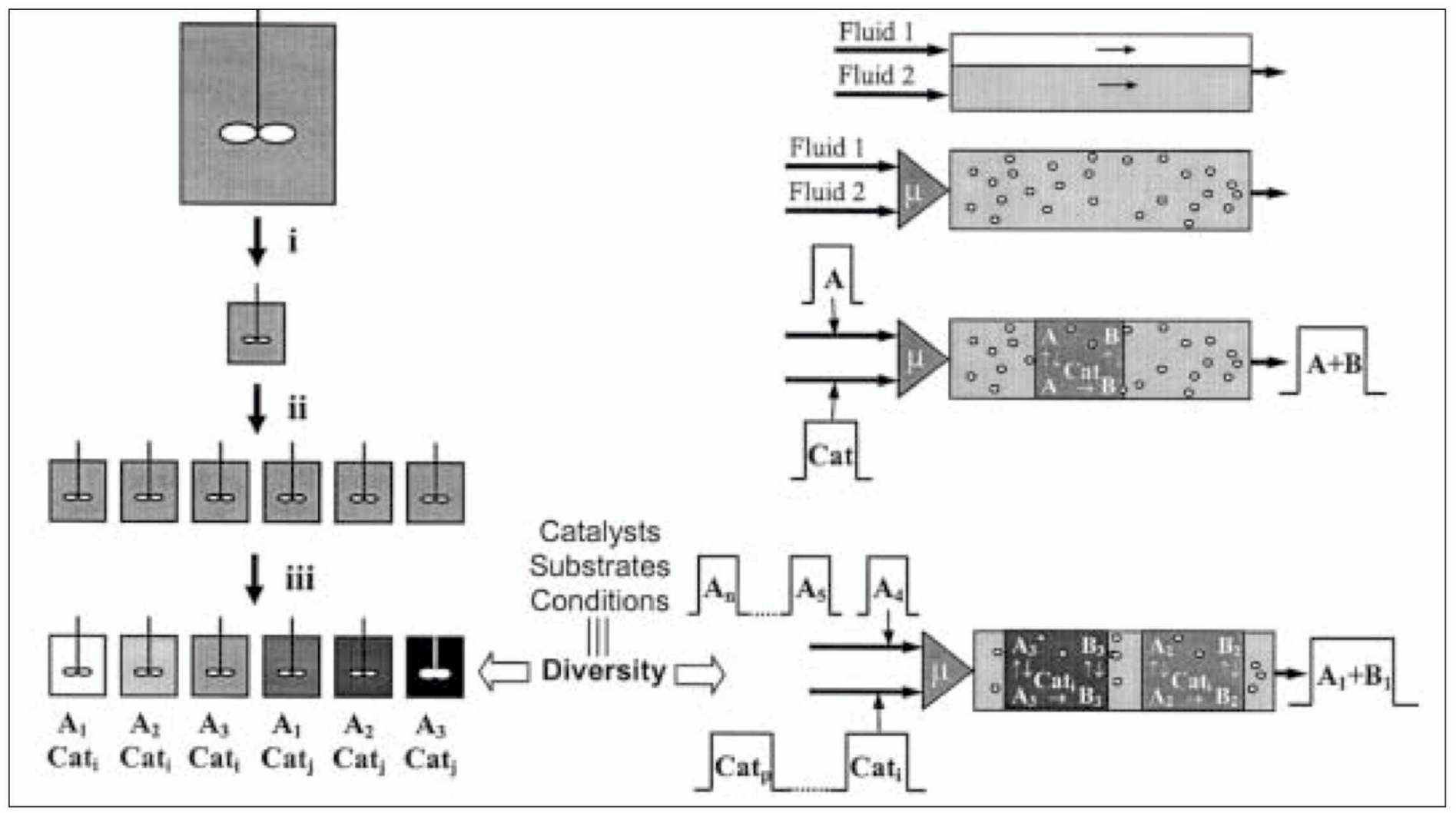

Fig. 1. Schematic representation of the reactor set-up for fast investigation of multiphase molecular catalysis illustrating two methods for the management of diversity. Left: parallel batch reactors. Right: sequential testing involving a micro-mixer and pulses. 


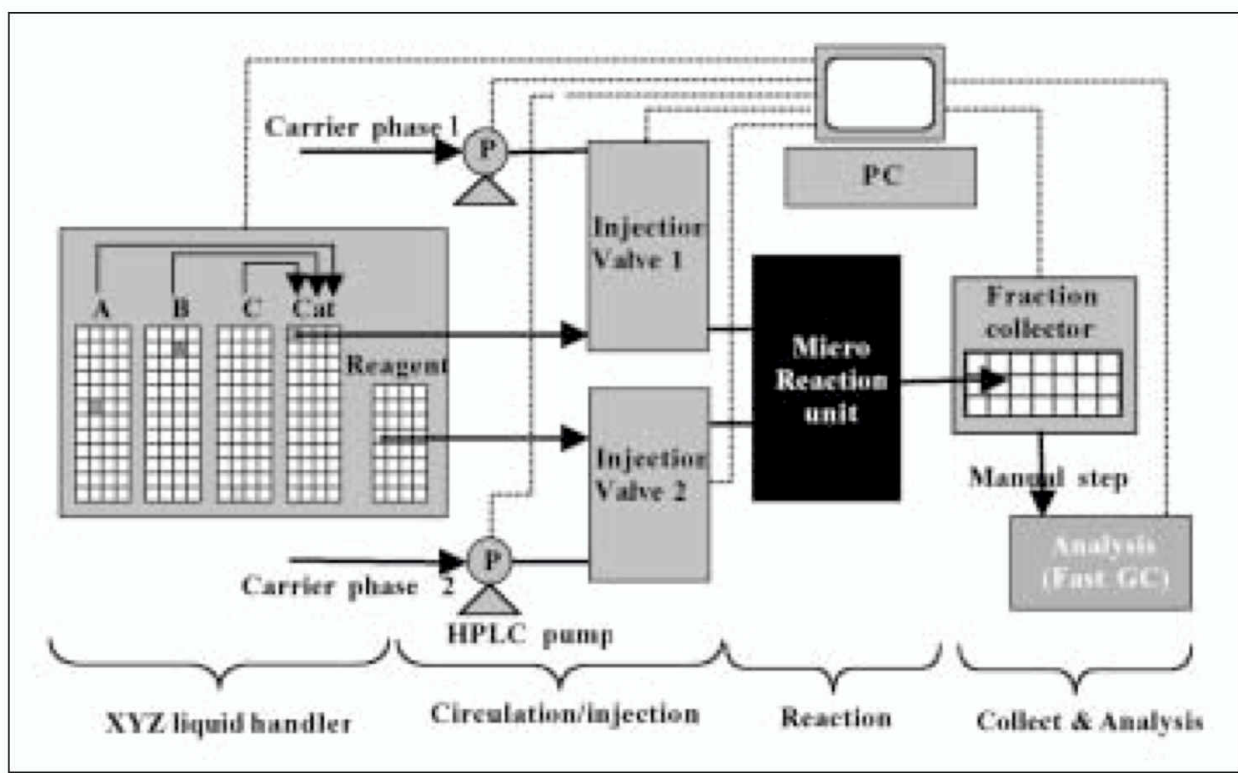

Fig. 2. Overall set-up used for HTS experiments. The liquid handler, pumps, injection valves, the collector and the GC apparatus are commercially available. The 'heart' of the system consists of modular interchangeable microreaction units for $L / L$ or $G / L$ reactions. Extension to other type of catalytic reactions, e.g. G/L/S is under progress.

Preliminary work has described the use of a micromixer for the high-throughput screening of $\mathrm{L} / \mathrm{L}$ and $\mathrm{G} / \mathrm{L}$ catalysis [9a]. The transformation of the basic, manually operated set-up used in this first report for the 'proof of principle' into a usable, unattended, and efficient apparatus for routine HTS is evaluated here.

The setting of the overall HTS apparatus, including automation, is described in Fig. 2. It is composed of a liquid handler, a reaction section and an analytical device. The liquid handler is used for both the preparation of catalysts and injection of catalyst/reagent into a reaction unit.

During operation, the microreaction unit is continuously fed with the carrier fluids. These can be two immiscible or miscible liquids or a gas (reacting gas such as $\mathrm{H}_{2}$ ) and a liquid. At periodic time intervals, pulses of the (liquid) catalyst to be screened and the substrate are injected. The pulses are then mixed in a micromixer [11] placed at the entrance of the microreaction unit. Alternatively, for longer residence times in $\mathrm{L} / \mathrm{L}$ catalysis, the $\mathrm{L} / \mathrm{L}$ emulsion is directed, via a programmable collector, into parallel small batch reactors arranged in a homemade rack.

\section{Results and Discussion}

\subsection{Monophasic Liquid Operations}

The ability of the automated liquid handler to operate with air-sensitive organometallic catalyst was evaluated first. The $\mathrm{H}$-transfer asymmetric reduction of acetophenone into $(R)$ - and $(S)$-phenylethanol in 2-propanol was choose as a test reaction (Scheme 1). Since mixing is not an issue for monophasic catalysis (the reaction is not so fast, $>1 \mathrm{~h}$ ), the microreaction unit depicted in Fig. 2 can be removed. In a first experiment, a library of 60 chiral ligands, covering 11 structural families, was combined with three metal precursors of $\mathrm{Ru}$ ( $[\mathrm{Ru}$ $\mathrm{Cl}_{2}(p$-cymene $\left.\left.)\right]_{2}\right), \mathrm{Rh}\left([\mathrm{RhCl}(\mathrm{cod})]_{2}\right)$ and $\mathrm{Ir}$ $(\operatorname{Ir}(\mathrm{acac})(\mathrm{cod}))$ since these metals are known to be active for this reaction. Selected examples of these ligands are pictured in Fig. 3.

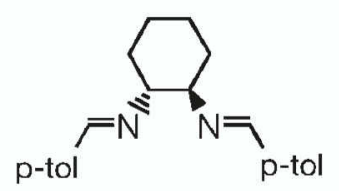

1<smiles>OCc1c(-c2ccc3ccc(F)c(C(F)(F)F)c3n2)ccc2cccnc12</smiles>

2<smiles>CC(C)[C@H](CO)N=CC1=Nc2c(O)cccc2CC1</smiles>

3

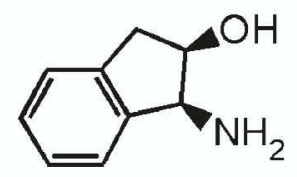

4<smiles>CNC1CCCC[C@H]1NC</smiles>

5

Fig. 3. Selected examples of ligands $L^{*}$ from the library used for evaluation in the H-transfer reduction of acetophenone. Some of the ligands (4 \& 5) are commercially available.

Thus, a chemical diversity number of $180 \mathrm{ligand} / \mathrm{metal}$ couples is achieved. However, it is also well known that reaction parameters such as the metal to ligand ratio, the substrate vs. metal concentration and co-catalyst (sodium isopropanolate)/metal are important. Hence, a total number of 1800 tests was performed/analyzed in less than ca. 20 days working time. Selected results are shown in Fig. 4, enantiomeric excess up to $86 \%$ have been obtained.

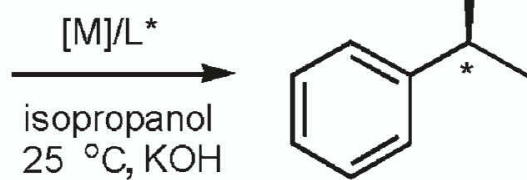




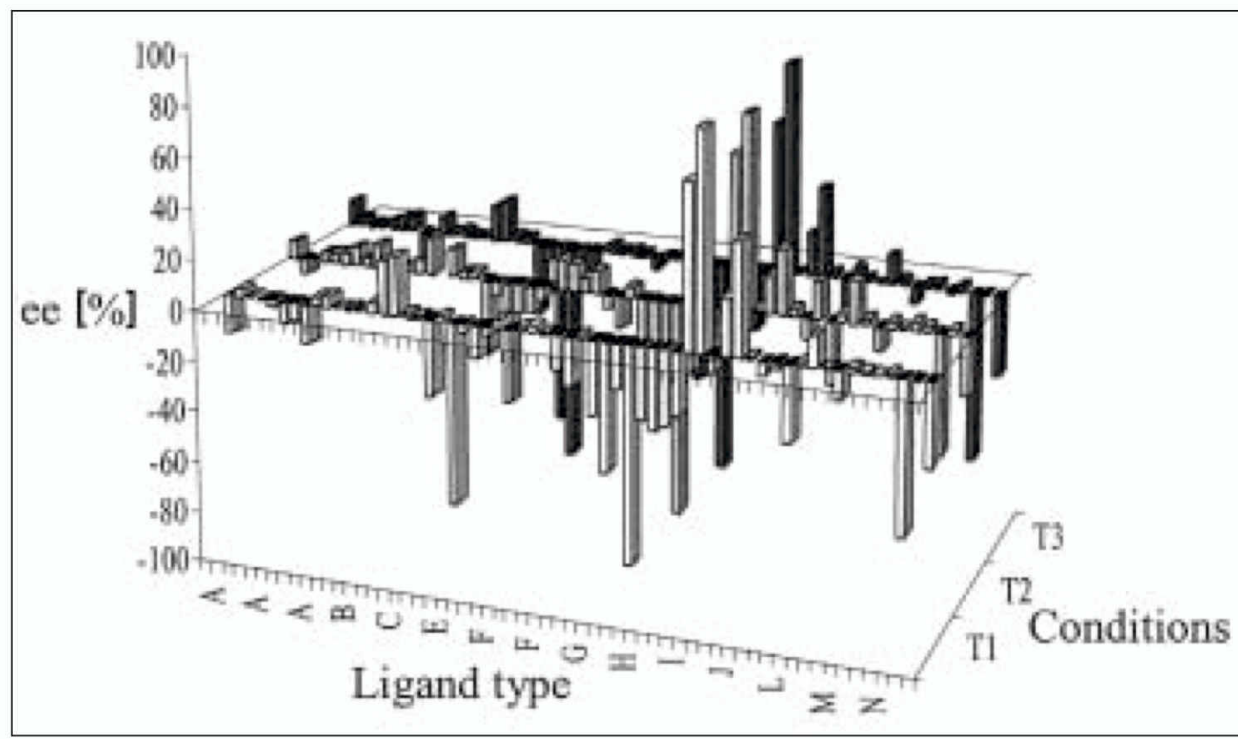

Fig. 4. Enantiomeric excess (\%) as a function of the ligand (A1....N6). The test was performed under different conditions T1, T2 \& T3 (T1: $[\mathrm{S}] /[\mathrm{M}]=25$, $[\mathrm{L}] /[\mathrm{M}]=1 ; \mathrm{T} 2:[\mathrm{S}] /[\mathrm{M}]=100,[\mathrm{~L}] /[\mathrm{M}]=$ 1; T3: $[\mathrm{S}] /[\mathrm{M}]=100,[\mathrm{~L}] /[\mathrm{M}]=2)$. Other conditions: $6 \mathrm{~h}, 20^{\circ} \mathrm{C}, 400 \mu \mathrm{l}, \mathrm{C}_{\mathrm{Ru}} 10^{-3} \mathrm{~mol}^{-\mathrm{I}^{-1}}, \mathrm{C}_{\mathrm{KOH}}$ $10^{-2} \mathrm{~mol} \mathrm{I}^{-1}$

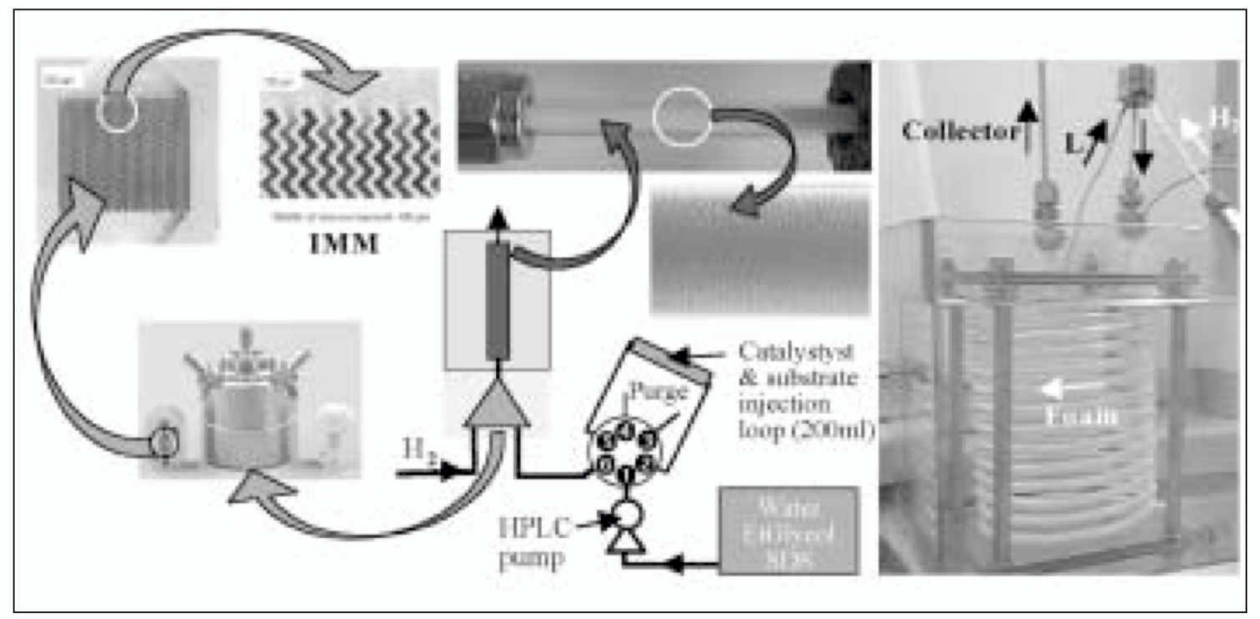

Fig. 5. Experimental set up for hydrogenation. The outlet is connected to an automated collector. The injection loop of the HPLC injection valve is connected to the liquid inlet $L$ shown on the picture. The coiled tube is placed in a temperature-controlled bath.

The exploitation of these first results point to the best catalytic systems being $\mathrm{Ru} / 4$ as reported in the literature. From these first experiments, a second library of ca. 20 ligands was designed. A second series of $c a .1400$ tests was performed which resulted in the discovery of a new class of ligands for this reaction that provides ees up to $93 \%$ with good activities. To summarize, the identification of both known and new catalytic systems for this monophasic catalysis reaction validates the use of the liquid handler for air-sensitive reactions.

\subsection{Biphasic Gas-Liquid Operations}

The 'microreaction unit' (see Fig. 2) for $\mathrm{G} / \mathrm{L}$ operation is described in Fig. 5. The range of operating conditions are pressure: 1-11 bar, temperature, $20-70^{\circ} \mathrm{C}$, residence time: $1-12 \mathrm{~min}$ for the straight $0.8 \mathrm{~m}$ reaction tube, 3-25 min for the coiled $3 \mathrm{~m}$ tube. Note that the nature of the material used for the tube, chosen for visual observation, limits the pressure range. Use of a stainless steel tube allows higher working pressures (50 bar). Under operation, the two fluid phases, i.e. the aqueous solution and hydrogen, flow continuously through the apparatus leading to a stable foam as observed in the tube. For a test, a pulse containing the substrate and the Rh/diphos catalyst dissolved in the water/ethylene glycol/SDS mixture is injected. This composition of the liquid is adjusted to obtain a good quality foam. The reagents are perfectly mixed in the micromixer leading to a reacting segment which travels along the tubular reactor. Collection at the outlet of the reactor, extraction of the organics and analysis afford conversion and ee data. Two parameters are of importance for the operability of the test: The stability of the gas-liquid foam and the size of the reacting segment. It has been found that the foam stability increases with the hydrogen pressure. This ensures residence times of up to $12 \mathrm{~min}$ at $70{ }^{\circ} \mathrm{C}$ without noticeable bubble coalescence. Analysis of the residence time distribution reveals some axial dispersion of the segment when the reacting tube is placed vertically, probably due to backmixing. That was neglected for the reactor/kinetic modeling and can be attenuated by placing the tube horizontally.

Using this set-up, the kinetics of the asymmetric hydrogenation of methyl- $Z$ acetamidocinamate (MAC) with a $[\operatorname{Rh}((S, S)$ BDPPTS $)]^{+}$water-soluble catalyst was studied (Scheme 2) [9c].

Control experiments have been performed in a specially designed mini batch reactor $\left(10 \mathrm{~cm}^{3}\right)$, optimized for low inventory of reacting solution, under the same conditions (liquid phase composition, temperature, etc.). The kinetic parameters have been computed from the hydrogen consumption and not from the (R) and (S) concentrations but it should in theory give the same results since $\mathrm{r}_{\mathrm{H} 2}=\mathrm{r}_{\mathrm{MAC}}=-\left(\mathrm{r}_{R}+\mathrm{r}_{S}\right)$ Comparison of the kinetic constant at 323 $\mathrm{K}, \mathrm{k}_{323}=9.3$ vs. $19.1\left(\mathrm{~m}^{3} \mathrm{kmol}^{-1} \mathrm{Mpa}^{-1}\right.$ $\mathrm{min}^{-1}$ ) and the activation energy is $31 \pm 4$ vs. $40 \pm 4\left(\mathrm{~kJ} \mathrm{~mol}^{-1}\right)$ in the micro-test and batch reactors respectively, indicated lower value in the microtest unit. This illustrates the difficulties to carry out experiments with a very low inventory of material. The data concerning the enantioselectivity are more in agreement, lying between 40 and $45 \%$.

\subsection{Biphasic Liquid-Liquid Operations}

The racemic or asymmetric isomerization of allylic alcohols into carbonyls with water-soluble catalysts in a biphasic heptane-water system has been investigated (Scheme 3).

To assess the efficiency of the micromixer, nine substrates with quite different solubilities in the catalytic aqueous layer have been tested in three set-ups. In the first set-up, the micromixer is not used, the mixing between the two phases is simply achieved by needle injection of the reagents and solvents (water, n-heptane, catalyst, and substrate) into small vials $\left(2 \mathrm{~cm}^{3}\right)$ in a home-made rack placed onto the liquid handler. In the second set-up, the micromixer was used as a pre-mixing unit, the 

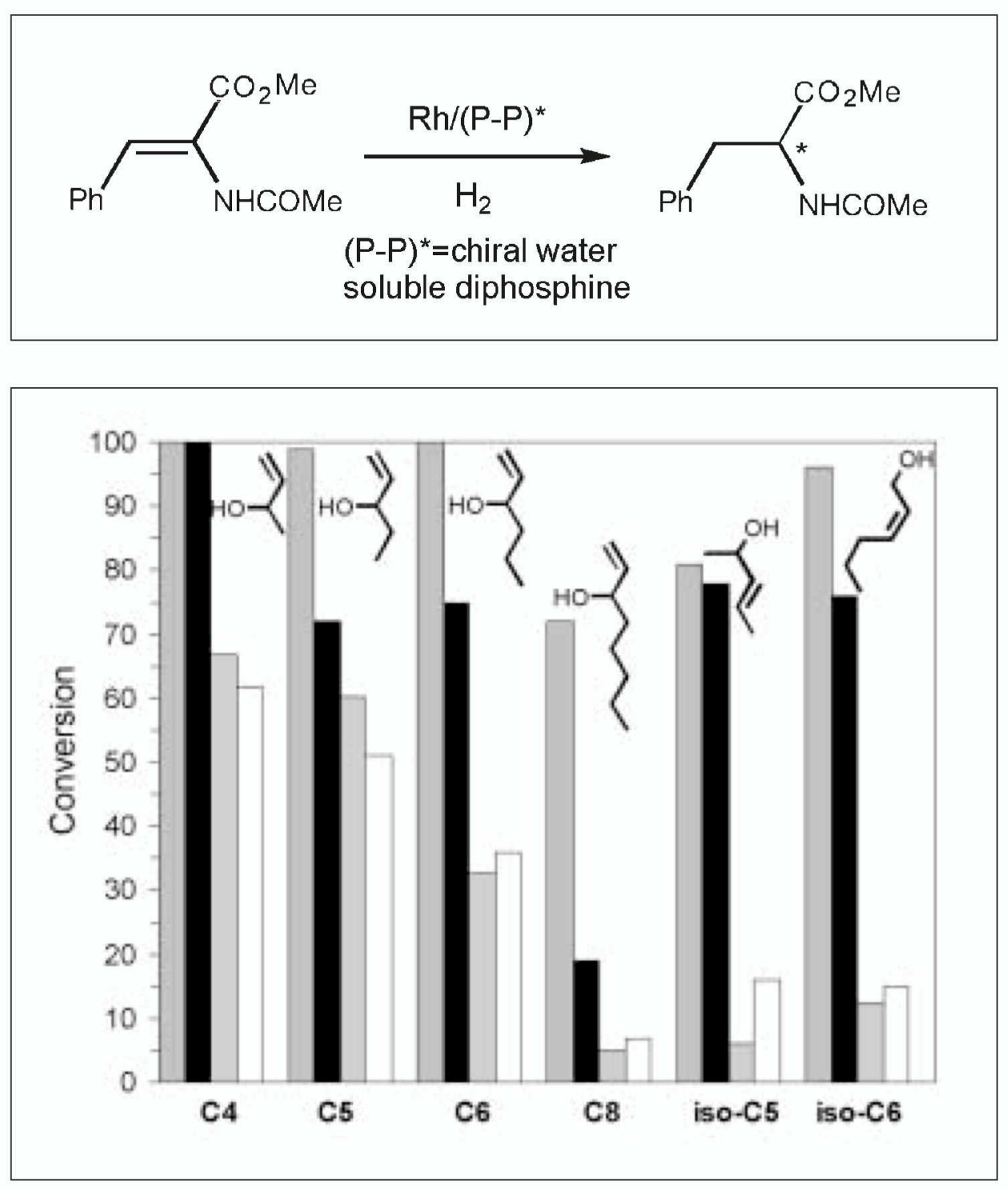

reacting emulsion being introduced as it was in the same vials for reaction. Note that in both these set-ups involving vials, the residence time was $1 \mathrm{~h}$. In a third set-up, a 'micrreaction unit' for liquid-liquid operation, similar to that used for G/L experiments (Fig. 5) was used. The gaseous fluid is replaced with a organic solvent (n-heptane) and the aqueous phase is now pure water. Indeed, with the help of the micromixer, there is no need of surfactant. The organic-water emulsion formed is very stable, up to several hours. The residence (reaction) time with the third set-up is 1-3 min. The results of the comparative study are displayed in Fig. 6. Thus except for the residence times, the other conditions, e.g. substrate and catalyst concentrations, were kept constant.

The comparison with experiments run in vials, with ( $\square$ ) and without ( $\square$ ) pre-mixing (i.e. with or without the micromixer) clearly demonstrate the advantage of using a micromixer. Conversions are significantly higher when using the micromixer, ex- cept for the readily soluble $\mathrm{C} 4$ for which mass transfer to the aqueous catalytic phase may not be an issue. However, for the long reaction time used for these vials experiments, the observed conversions are quite high, irrespective of the substrates, which cannot allow the ranking of reactivity between the different substrates. With the third set-up ( $\square$ ), however, since the reaction occurs in the segment traveling in the reaction tube, short reaction times can be accurately controlled and quantitative data for e.g. ranking of the substrate reactivity or kinetic measurements can be obtained. For example, the decrease in conversion for $\mathrm{C} 4$ through $\mathrm{C} 8$ simply reflects the difference in solubility, i.e. an observation of the properties of the molecules and not the signature of the apparatus. Intrinsic chemical reactivities are actually observed with the third set-up which is demonstrated by the lower conversions observed for normal vs. iso-allylic alcohols (C5 and C6 vs. iso-C5 and iso-C6). Indeed, it is well known that internal $\mathrm{C}-\mathrm{C}$ double bonds isomerize much

Scheme 2 .

Fig. 6. Comparison of the biphasic isomerization of some allylic alcohols with four different reactors: reaction in vials, with $(\square)$ and without $\square$ the micromixer; reaction in a set-up combining the micromixer and a reaction tube $(\square)$ (similar to that described in Fig. 5) and in a mini-batch reactor of $10 \mathrm{~cm}^{3}(\square)$. Conditions 50 ${ }^{\circ} \mathrm{C}, \mathrm{C}_{\mathrm{Rh}} 0.011 \mathrm{kmol} . \mathrm{m}^{-3}, \mathrm{~S} / \mathrm{Rh} 100$, n-heptane. Residence time for ( $\square$ and $\square$ ), $1 \mathrm{~h}$; for ( $\square$ and 口), $100 \mathrm{~s}$.

more slowly. Finally, data obtained in a well-mixed mini-batch reactor $\left(10 \mathrm{~cm}^{3}\right)$ are also displayed $(\square)$. The good agreement between data obtained with the third set-up and that from the mini-batch further demonstrates the validity of this concept. This qualitative study demonstrates the need of the micromixer to reach intrinsic chemical properties in liquid-liquid HTS.

\section{Conclusion}

The following table provides features that have been either achieved or that are achievable for selected liquid-liquid and gas-liquid experiments (Table). It also provides a rough comparison with traditional albeit optimized (mini-batch) laboratory reactors according to the criteria discussed in the introductory section.

These features must be considered as a first evaluation of the potential use of microdevices for HTS. The gain in quantity of sample required, in testing throughput and 


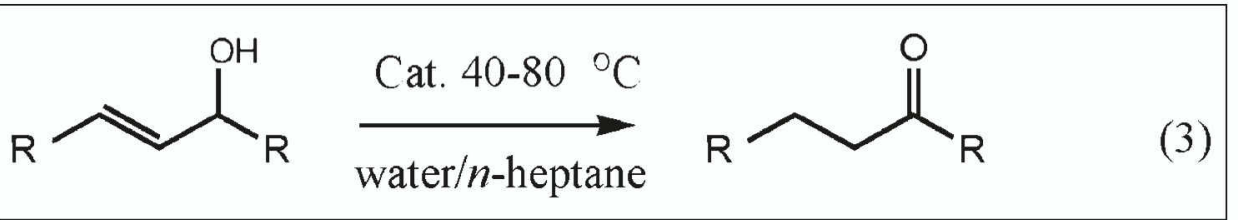

Table. Comparison of the performance of a traditional mini-batch reactor with the microreaction units described in this work for $L / L$ and $G / L$ reactions.

\begin{tabular}{|c|c|c|c|}
\hline Feature & Mini-batch & Micro L/L & Micro G/L \\
\hline Reaction volume $\left[\mathrm{cm}^{3}\right]$ & 10 & 0.2 & 0.1 \\
\hline Average amount of Rh per experiment $[\mu \mathrm{g}]$ & $500-1000$ & $10-80$ & $5-20$ \\
\hline Typical amount of ligand per exp. [umol] & 10 & 0.5 & 0.1 \\
\hline Temperature range $\left[{ }^{\circ} \mathrm{C}\right]$ & $20-100$ & $20-70$ & $20-80$ \\
\hline Pressure range [bar] & $1-100$ & - & $1-11^{\mathrm{a}}$ \\
\hline Residence time [min] & $>10$ & $1-120$ & $1-30$ \\
\hline Average $T \mathrm{TF}^{\mathrm{b}}$ achieved during study $\left[\mathrm{d}^{-1}\right]$ & 2 & 40 & 15 \\
\hline Maximum actually achievable $\Pi \mathrm{TF}\left[\mathrm{d}^{-1}\right]$ & 3 & 50 & $40^{c}$ \\
\hline Range of solvents (viscosity, density,...) & large & large & restricted ${ }^{d}$ \\
\hline Automation of reagents/catalysts injection & No & Yes & Yes \\
\hline Automation of sample collection & No & e & Yes \\
\hline
\end{tabular}

a Limitation depends on the material used for the reacting tube (quartz).

b TTF is the throughput testing frequency i.e. the number of experiments per day.

c Delay time between injection in the micro-test is only 3-5 min but both the optimised chiral GC analysis (one run per $12 \mathrm{~min}$ ) and the restriction of unattended operations to day time limit the test throughput.

d Only aqueous phase containing a foaming agent and a viscosity agent can be used.

${ }^{\text {e }}$ Not yet performed.

in automation will increase. While this first approach was based on a concept involving mixing, the design of new microdevices based on other concepts, e.g. contact with falling film reactors, will overcome the bottlenecks presented here.

Integration of analytics is also a challenge. Traditional techniques such as GC or HPLC, although outstanding microcolumns have been developed leading to retention time of only a few minutes for complex molecules, are limited for HTS. New flow-through micro-devices for chiral analysis that can be cheaply integrated to micro-HTS reactors are needed.

\section{Acknowledgements}

Funding from the European Commission (GRD-2000-256262) through the KEMICC (Key Elements for the application of Microreactors to Catalytic Chemistries). The CNRS and the Ecole Supérieure de Chimie Physique Electronique de Lyon is acknowledged. The $\mathrm{PhD}$ of N.P. is supported by a grant of the French minister of education. This work would not have been possible without the contribution of Prof. Olivier Riant, University of Louvain-la-Neuve, Belgium, and Dr. Volker Hessel, Institute for Micromechanics Mainz, Germany.
[1] See for example: a) B. Jandeleit, D.J. Schaefer, T.S. Powers, H.W. Turner, W.H. Weinberg, Angew. Chem. Int. Ed. 1999, 38, 2494; b) P.P. Pescarmona, J.C. van der Waal, I.E. Maxwell, T. Maschmeyer, Catal. Letters 1999, 63, 1; c) S. Senkan, Angew. Chem. Int. Ed. 2001, 40, 312; d) Special issue Catal. Today 2001, 67.

[2] See: 'Applied homogeneous catalysis with organometallic compounds', Eds. B. Cornils, W.A. Herrmann, VCH, Weinheim, 1996, vol. $1 \& 2$.

[3] a) R. Noyori, 'Asymmetric Catalysis in Organic Synthesis', Wiley, New York, 1994, pp 33; b) J.M. Brown (chap. 5.1.), R.L. Halterman (chap. 5.2.), T. Ohkuma, R. Noyori (chap. 6.1.) and H.U. Blaser, F. Spindler (chap. 6.2.) in 'Comprehensive Asymmetric Catalysis', Eds. E.N. Jacobsen, A. Pfaltz, H. Yamamoto, Springer, Berlin, 1999.

[4] M.T. Reetz, Angew. Chem. Int. Ed. 2001, 40, 284.

[5] A review of laboratory reactors for heterogeneous catalysis can be found in: F. Kapstein, J.A. Mouljin, in 'Handbook of Heterogeneous Catalysis', Eds. G. Ertl, H. Knözinger, J. Weitkamp, Wiley-VCH, Weinheim, 1997, chap. 9, pp 1359.

[6] a) Y. Sun, R.N. Landau, J. Wang, C. Le Blond, D.G. Blackmond, J. Am. Chem. Soc. 1996, 118, 1348; b) C. de Bellefon, D. Schweich, N. Tanchoux, Récents Prog. Genie Procédés 1999, 13, 237.
[7] a) W. Ehrfeld, V. Hessel, H. Löwe, 'Microreactors', Wiley-VCH, Weinheim, 2000; b) A. Gravilidis, P. Angeli, E. Cao, K.K. Yeong, Y.S.S. Wan, Trans. IChemE. 2002, 80, 3; c) P.D.I. Fletchar, S.J. Haswell, E. Pombo-Villar, B.H. Warrington, P. Watts, S.Y.F. Wong, H. Zhang, Tetrahedron 2002, 58, 4735.

[8] For a short review of parallel multi-batch HTS apparatus for gas-liquid catalysis, see: C. de Bellefon in 'Principles and Methods for Accelerated Catalyst Design and Testing', Eds. E. Derouane, V. Parmon, F. Lemos, F. Ramôa Ribeiro, NATO Science Series, Kluwer, Dordrecht, 2002 , pp 71 .

[9] a) C. de Bellefon, N. Tanchoux , S. Caravieilhes, P. Grenouillet, V. Hessel, Angew. Chem. Int. Ed. 2000, 39, 3442; b) C. de Bellefon, S. Caravieilhes, P. Grenouillet in 'Microreaction Technology', Eds. M. Matlosz, W. Ehrfeld, J.P. Baselt, Springer, 2002, 408; c) C. de Bellefon, N. Pestre, T. Lamouille, P, Grenouillet, $A d v$. Synth, Catal. 2003, in press.

[10] H. Pennemann, V. Hessel, IMM Mainz, Germany, personal communications.

[11] For further information on the micromixer used in this work, see: a) W. Ehrfeld, K. Golbig, V. Hessel, H. Löwe, Th. Richter Ind. Eng. Chem. Res. 1999, 38, 1075 ; b) H. Löwe, W. Ehrfeld, V. Hessel, T. Richter, J. Schiewe, in 'Proceedings of IMRET 4', March 2000, Atlanta, USA. 